\title{
MODELAGEM LINEAR GENERALIZADA PARA DESCREVER O INCREMENTO EM ÁREA TRANSVERSAL DE ÁRVORES INDIVIDUAIS DE IMBUIA
}

\author{
Veridiana Padoin Weber ${ }^{*}$, César Augusto Guimarães Finger ${ }^{2}$, Emanuel Arnoni $\operatorname{Costa}^{2}$, Anna Paula Lora \\ Zimmermann², Régis Villanova Longhi ${ }^{3}$ \\ 1* Universidade Tecnológica Federal do Paraná, Dois Vizinhos, Paraná, Brasil - veridianapadoin@utfpr.edu.br; \\ ${ }^{2}$ Universidade Federal de Santa Maria, Santa Maria, Rio Grande do Sul, Brasil - cesarfinger.ufsm@ gmail.com, emanuelarnonicost@ hotmail.com; \\ zimmermann-a@hotmail.com; \\ ${ }^{3}$ Universidade Federal de Alagoas, Maceió, Alagoas, Brasil - regis.longhi@icbs.ufal.br
}

Recebido para publicação: 07/08/2017 - Aceito para publicação: 31/01/2018

\begin{abstract}
Resumo
Modelos de crescimento em nível de árvores individuais são aplicados para a quantificação e descrição dos fatores que afetam o ritmo de crescimento das árvores. Nesse sentido, o presente estudo objetivou aplicar a abordagem de Modelos Lineares Generalizados (MLG) para descrever o incremento periódico anual em área transversal de árvores individuais de Ocotea porosa (Nees) Barroso em floresta natural mista e inequiânea a partir de medições de variáveis dendrométricas e morfométricas e de índices de competição. Na modelagem, avaliaram-se três tipos de distribuições aleatórias contínuas para a variável resposta: Normal, Gamma e Gaussiana Inversa; com diferentes funções de ligação: identidade $(\mu)$, logarítmica $(\ln \mu)$ e de potência $\left(\mu^{\lambda}\right)$. $O$ MLG com distribuição Gamma e função de ligação logarítmica apresentou as melhores estimativas, sendo o crescimento da espécie expresso pelo diâmetro, altura total, diâmetro de copa, relação h/d e pelo índice de competição de Lorimer. Essa metodologia permitiu predições não enviesadas, com maior acuracidade e realismo biológico na interpretação dos coeficientes de regressão, quando comparados aos métodos convencionais com transformação na variável dependente.
\end{abstract}

Palavras-chave: Floresta Ombrófila Mist, Ocotea porosa, crescimento, modelo generalizado.

\begin{abstract}
Generalized linear modeling to describe the increment of basal area of individual trees of imbuia. Growth models on level of individual tree are applied for the quantification and description of the factors that affect the growth rate of the trees. The present study aimed to apply the approach of Generalized Linear Models (MLG) to describe the periodic annual increment in basal area of individual trees of Ocotea porosa (Nees) Barroso in natural mixed and uneven-aged forests, considering the measurements of dendometric and morphometric variables, and the competition index. We evaluated three types of modeling of continuous random distributions for a response variable: Normal, Gamma, and Inverse Gaussian; with different link functions: identity $(\mu)$, logarithmic $(\ln \mu)$, and power $\left(\mu^{\lambda}\right)$. MLG with Gamma distribution and logarithmic link function had the best estimates. The growth of the species was expressed by diameter, total height, crown diameter, $\mathrm{h} / \mathrm{d}$ relation, and Lorimer competition index. This methodology allowed non-skewed predictions, with greater accuracy, and biological realism in the interpretation of the regression coefficients, when compared to conventional methods of transformation in the dependent variable.
\end{abstract}

Keywords: Mixed Ombrophilous Forest, Ocotea porosa, growth; generalized model.

\section{INTRODUÇÃO}

O conhecimento dos padrões de crescimento da floresta e fatores que afetam o incremento é fundamental para que pesquisadores e gestores possam avaliar e aperfeiçoar práticas silviculturais (LHOTKA, 2017). No caso das florestas equiâneas, os modelos de crescimento servem como auxílio no entendimento dos fatores que influenciam no crescimento, embora o incremento seja uma função da média das variáveis analisadas (CAMPOS; LEITE, 2013). Em florestas naturais, devido à presença de diferentes espécies e dimensões que possuem exigências distintas por nutrientes, luz e espaço, a quantificação do crescimento é bem mais complexa quando comparada à de uma floresta pura (KIERMAN et al., 2008).

Os modelos de crescimento em nível de árvore individual preveem mudanças ao longo do tempo com base nas relações entre as taxas de incremento e os atributos dendrométricos, dimensão da copa, nível de competição da árvore, objetivo em relação aos seus competidores e, ainda, qualidade do sítio

FLORESTA, Curitiba, PR, v. 48, n. 1, p. 123 - 132, jan. / marc. 2018

Weber. V. P. et al.

ISSN eletrônico $1982-4688$

DOI: $10.5380 / \mathrm{rf} . v 48$ i1.54368 
(CUNHA et al., 2016). Dentre as vantagens do uso dos modelos de árvores individuais está a sua flexibilidade, a possibilidade de analisar inúmeras variáveis para caracterizar o crescimento e o fornecimento de resultados detalhados, de acordo com a necessidade da pesquisa (WEISKITTEL et al., 2011). Devido a esses benefícios, diversos autores têm se utilizado dessa técnica para descrever e conhecer os fatores mais influentes sobre o crescimento de espécies florestais (COSTA et al., 2015; ZIMMERMANN et al., 2016).

Dentre as variáveis influentes no crescimento, as características morfométricas, o espaço vital e a competição sofrida pela árvore são parâmetros fundamentais para o manejo de árvores individuais, no qual o principal objetivo é fornecer condições ideais para o crescimento das árvores, potencializando seu incremento (COSTA et al., 2015). O nível de competição dado pelo espaço entre as árvores é o principal fator na limitação do crescimento, podendo ser medido por meio de índices de competição (CANETTI et al., 2016) dependentes e independentes da distância. Esses índices não descrevem todos os processos biológicos complexos envolvidos na competição, mas fornecem medições dos resultados desses processos e demonstram ser úteis na previsão do crescimento das árvores (DANIELS et al., 1986).

Entretanto, muitas vezes, os dados de crescimento e variáveis dimensionais das árvores em florestas naturais não seguem um padrão de distribuição normal. Nesses casos, distribuições mais flexíveis para a variável resposta permitem obter ganho na performance preditiva do modelo. Assim, a abordagem de modelos lineares generalizados (MLG) torna-se uma ferramenta estatística de grande potencialidade de aplicação. Segundo Paula (2013), esse método possibilita aumentar as opções da distribuição da variável resposta e dar maior flexibilidade com uso de uma função de ligação, que faz a relação da variável resposta e seu preditor linear.

Ocotea porosa (Nees) Barroso (imbuia) desempenhou um papel fundamental no passado quanto ao desenvolvimento econômico e cultural da região Sul do Brasil. Estudos recentes, como os de Mattos et al. (2010) em florestas naturais e Santos et al. (2015) em povoamentos homogêneos, apresentam o ritmo de crescimento da espécie a partir de avaliações dendrocronológicas. Entretanto, há carência de pesquisas com a espécie que busquem compreender a influência da qualidade do sítio, das variáveis de tamanho e forma da copa, do status competitivo da árvore objetivo e suas influências no que tange ao crescimento e produção em florestas naturais inequiâneas.

Diante disso, este estudo tem como objetivo descrever o incremento em área transversal de árvores individuais de $O$. porosa em floresta natural mista e inequiânea na Região Central do estado de Santa Catarina, a partir de variáveis dendrométricas e morfométricas e de índices de competição, aplicando-se o conceito de modelo linear generalizado.

\section{MATERIAL E MÉTODOS}

O estudo foi realizado nos municípios de Calmon e Caçador, ambos localizados no estado de Santa Catarina, em uma altitude média de 1100 metros. A região onde se situam os municípios caracteriza-se por apresentar clima do tipo $\mathrm{Cfb}$, de acordo com a classificação climática de Köppen, temperatura média anual entre 15 e $16{ }^{\circ} \mathrm{C}$, variando entre $29,3{ }^{\circ} \mathrm{C}$ e $4,2^{\circ} \mathrm{C}$. As chuvas são distribuídas em todos os meses do ano, com precipitação anual em torno de $1730 \mathrm{~mm}$ (ALVARES et al., 2013).

A vegetação pertence à tipologia Floresta Ombrófila Mista (INSTITUTO BRASILEIRO DE PESQUISA E ESTATÍSTICA, 2012), sendo predominante a presença de Araucaria angustifolia (Bertol.) Kuntze (Pinheirobrasileiro) no estrato superior da floresta em associação a Ocotea porosa.

A amostragem intencional de 122 árvores individuais de $O$. porosa foi realizada com vista a cobrir toda a amplitude de distribuição diamétrica encontrada na floresta natural dos locais estudados. Esse procedimento de amostragem também foi realizado por Costa et al. (2016a) para obter árvores representativas para o estudo de relações morfométricas. Cada árvore objetivo foi considerada um ponto amostral e teve suas concorrentes mensuradas, sendo consideradas aquelas cujas copas encobriam ou tocavam a copa da árvore objetivo. Não houve um número fixo de árvores concorrentes medidas, dado o comportamento irregular das árvores em florestas mistas inequiâneas.

Os atributos das árvores objetivo medidos foram: circunferência à altura do peito, com fita métrica, que foi transformada em diâmetro à altura do peito (d); altura total (h), altura comercial (hc), considerada até o ponto de máximo aproveitamento do fuste, e altura de inserção da copa (hic), medidas com hipsômetro Vertex III; comprimento de copa (cc), calculado pela diferença entre a altura total e a altura de inserção da copa; diâmetro de copa (dc), calculado pelo dobro da média aritmética de oito raios de copa mensurados com o hipsômetro Vertex III (função DME) e bússola para orientar nas direções dos pontos cardeais; área de projeção horizontal de copa (APHC), considerando área circular; e número de árvores por hectare (N.ha $\left.{ }^{-1}\right)$, calculado pela razão entre um hectare e a área de projeção horizontal de copa de cada árvore individual de O. porosa.

A partir dessas variáveis, foram derivados índices morfométricos: percentagem de copa $(\mathrm{Pc})=(\mathrm{cc} / \mathrm{h}) * 100$; índice de abrangência $(\mathrm{Ia})=\mathrm{dc} / \mathrm{h}$; índice de saliência $(\mathrm{Is})=\mathrm{dc} / \mathrm{d}$; formal de copa $(\mathrm{Fc})=\mathrm{dc} / \mathrm{cc}$ e relação h/d. Das 
árvores consideradas concorrentes da árvore objetivo, foram medidas as variáveis diâmetro à altura do peito (d); altura total (h); e distância horizontal entre a árvore objetivo $\left(\mathrm{L}_{\mathrm{ij}}\right)$, e calculados índices de competição de acordo com as dimensões da árvore objetivo e suas vizinhas concorrentes (Tabela 1).

Tabela 1. Índices de competição calculados para $O$. porosa.

Table 1. Competition indices calculated for $O$. porosa.

\begin{tabular}{cl}
\hline Índice de competição & \multicolumn{1}{c}{ Expressão } \\
\hline Basal Area Larger (IC1) & $=\Sigma\left(\pi \cdot \mathrm{d}_{\mathrm{j}}^{2} / 40000\right)$ \\
\hline Glover e Holl I (IC2) & $=\left(\mathrm{h}_{\mathrm{i}}^{2} / \overline{\mathrm{h}}_{\mathrm{j}}^{2}\right)$ \\
\hline Glover e Holl II (IC3) & $=\Sigma\left(\mathrm{d}_{\mathrm{i}}^{2} / \overline{\mathrm{d}}_{\mathrm{j}}^{2}\right)$ \\
\hline Lorimer (IC4) & $\mathrm{d}_{\mathrm{i}}$ \\
\hline Hegyi (IC5) & $=\Sigma\left(\mathrm{d}_{\mathrm{i}} / \mathrm{d}_{\mathrm{j}}\right)\left(1 / \mathrm{L}_{\mathrm{ij}}\right)$ \\
\hline Rouvinen e Kuuluvainen (IC6) & $=\Sigma\left(\mathrm{d}_{\mathrm{j}} / \mathrm{L}_{\mathrm{ij}}\right)$
\end{tabular}

Em que: $\mathrm{d}=$ diâmetro à altura do peito; $\mathrm{h}=$ altura total $; \mathrm{i}=$ árvore objeto; $\mathrm{j}$ = árvore competidora; $\mathrm{L}_{\mathrm{ij}}=$ distância entre a árvore (i) e competidoras (j); IC1-IC6 = índices de competição de 1 a 6 , respectivamente.

Para a análise do crescimento de $O$. porosa, foram retirados dois rolos de incremento com o Trado de Pressler na altura do diâmetro à altura do peito em posição de $90^{\circ}$ entre si. Os anéis foram identificados, marcados e mensurados no sistema LINTAB. A média entre os dois rolos foi calculada para a determinação do incremento periódico anual em área transversal (IPAg), considerando os últimos cinco anéis de crescimento. Esse período tem sido utilizado por diversos autores para descrever a influência dos fatores morfométricos, dendrométricos e de competição sobre o crescimento, uma vez que pode-se considerar que, nesse intervalo de tempo, as dimensões da copa das árvores não sofreram modificações expressivas (COSTA et al., 2016b).

$$
\mathrm{IPA}_{\mathrm{g}}=[(\mathrm{g}-\mathrm{g}-5 \mathrm{t}) / 5]
$$

em que: $\mathrm{IPA}_{\mathrm{g}}=$ incremento periódico anual em área transversal, em $\mathrm{cm}^{2} \cdot \mathrm{ano}^{-1} ; \mathrm{g}=$ área transversal, em $\mathrm{cm}^{2}, \mathrm{no}$ ano atual; $\mathrm{g}_{-5 \mathrm{t}}=$ área transversal, em $\mathrm{cm}^{2}$, nos últimos cinco anos

A análise de correlação de Pearson foi realizada entre o IPAg e todas as variáveis dendrométricas, morfométricas e os índices de competição para verificar o grau de associação entre as variáveis. Para a etapa de modelagem, foram selecionadas variáveis independentes de maior correlação com a variável resposta (IPAg), sendo dada preferência para as que apresentassem valor de explicação biológica, como os atributos dimensionais de copa, de concorrência e de sítio.

Um modelo linear generalizado (MLG) é composto por três componentes básicos: um componente aleatório, um preditor linear e uma função de ligação (FOX, 2015). Para a distribuição condicional da variável resposta, foram testadas as distribuições aleatórias contínuas Normal, Gamma e Gaussiana inversa. As funções de ligação avaliadas foram: identidade $(\mu)$, logarítmica $(\ln \mu)$ e de potência $\left(\mu^{\lambda}\right)$. A qualidade dos ajustes foi analisada segundo a distribuição a que os dados se ajustaram conjuntamente com a respectiva função de ligação. A metodologia utilizada para a modelagem linear generalizada foi baseada nas publicações de Cordeiro e Demétrio (2008) e Paula (2013).

A estimativa dos coeficientes de regressão para o modelo de IPAg foi realizada pelo método de máxima verossimilhança. A significância e o ajustamento do modelo foram medidos pelos valores dos critérios de informação de Akaike (AIC), critério de informação Bayesiano (BIC) e a dispersão gráfica dos resíduos de Pearson em função dos valores IPAg estimados. O teste de Wald foi utilizado como teste de hipótese para observar a significância dos coeficientes da equação.

FLORESTA, Curitiba, PR, v. 48, n. 1, p. 123 - 132, jan. / marc. 2018

Weber. V. P. et al.

ISSN eletrônico $1982-4688$

DOI: $10.5380 /$ rf.v48 i1.54368 
As análises estatísticas foram realizadas com o pacote estatístico SAS v. 9.2 (SAS INSTITUTE, 2011). Para avaliar a correlação de Pearson entre as variáveis, foi usado o procedimento PROC CORR. Estatísticas descritivas das variáveis foram obtidas com o PROC MEANS. A modelagem do IPAg foi realizada pelo método de máxima verossimilhança com o PROC GENMOD. A manipulação de dados e desenvolvimento de gráficos foi realizado com a planilha eletrônica Excel.

\section{RESULTADOS}

Foram mensuradas 122 árvores individuais de $O$. porosa, distribuídas em sete classes de diâmetro com intervalo de $10 \mathrm{~cm}$ entre si e amplitude diamétrica variando de 10,2 a 78,3 (Figura 1).

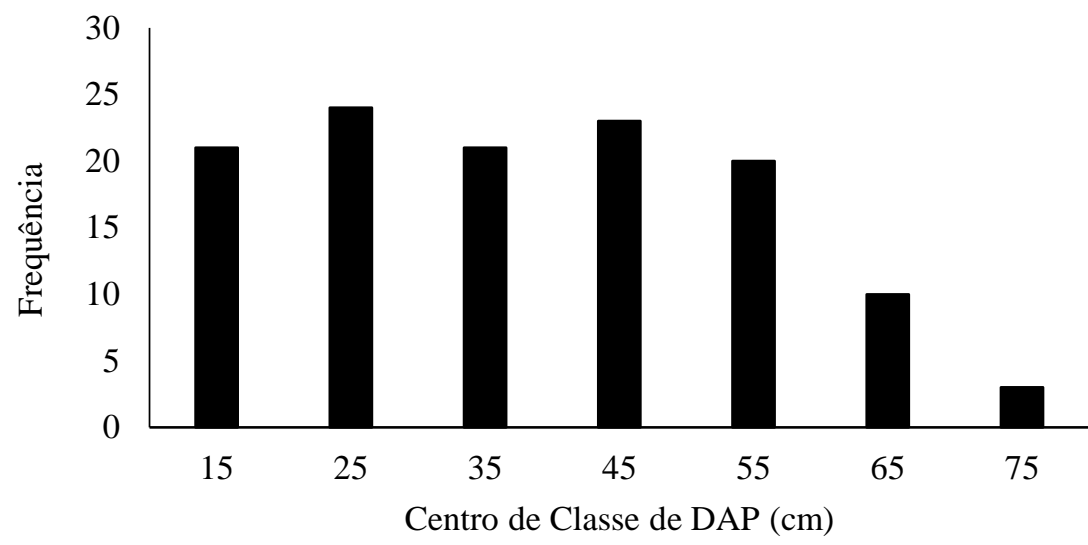

Figura 1. Distribuição de frequência por centro de classe diamétrica das árvores amostradas de O. porosa.

Figure 1. Frequency distribution by diameter class center of the sampled trees of $O$. porosa.

A altura total média das árvores foi de $17,4 \mathrm{~m}$, variando entre 9,8 e 26,3 m. O incremento periódico anual em área transversal médio foi de $25,21 \mathrm{~cm}^{2}$.ano ${ }^{-1}$, e o incremento em diâmetro médio de $0,43 \mathrm{~cm}$.ano ${ }^{-1}$, este variando de $0,05 \mathrm{~cm} . \mathrm{ano}^{-1}$ a $1,03 \mathrm{~cm} \cdot \mathrm{ano}^{-1}$ (Tabela 2 ).

Tabela 2. Estatística descritiva e correlação de Pearson entre o incremento periódico em área transversal de $O$. porosa e suas variáveis dendrométricas e morfométricas e índices de competição.

Table 2. Pearson correlation between the annual periodic increment in basal area of O. porosa and its dendrometric and morphometric variables, and competitions indices.

\begin{tabular}{|c|c|c|c|c|c|c|c|c|}
\hline \multirow{2}{*}{ Características } & \multirow{2}{*}{ Variável } & \multirow{2}{*}{ Mín. } & \multirow{2}{*}{ Média } & \multirow{2}{*}{ Máx. } & \multirow{2}{*}{$\mathbf{S}$} & \multirow{2}{*}{ CV\% } & \multicolumn{2}{|c|}{ IPAg $\left(\mathrm{cm}^{2} \cdot\right.$ ano $\left.^{-1}\right)$} \\
\hline & & & & & & & $\rho^{*}$ & Prob. \\
\hline \multirow{3}{*}{ Incremento } & IPAd & 0,05 & 0,43 & 1,03 & 0,26 & 59,3 & - & - \\
\hline & IPAg & 0,86 & 25,21 & 91,19 & 19,48 & 77,2 & - & - \\
\hline & $\mathrm{IPAg} \%$ & 0,36 & 2,63 & 9,77 & 1,94 & 73,9 & - & - \\
\hline \multirow{6}{*}{ Dendrométricas } & $\mathrm{d}$ & 10,2 & 37,4 & 78,3 & 16,8 & 45,1 & 0,64 & $<0,0001$ \\
\hline & $\mathrm{d}_{-5 \mathrm{t}}$ & 9,3 & 35,2 & 77,2 & 16,7 & 47,5 & 0,58 & $<0,0001$ \\
\hline & $\mathrm{d}_{\mathrm{sc}}$ & 9,7 & 35,2 & 75,2 & 15,9 & 45,1 & 0,64 & $<0,0001$ \\
\hline & $\mathrm{h}$ & 9,8 & 17,4 & 26,3 & 3,8 & 21,9 & 0,50 & $<0,0001$ \\
\hline & hic & 3,0 & 10,1 & 17,3 & 2,7 & 27,1 & 0,28 & 0,0019 \\
\hline & hc & 2,1 & 7,7 & 13,3 & 2,3 & 29,5 & 0,13 & 0,1438 \\
\hline \multirow{4}{*}{ Morfométricas } & $\mathrm{cc}$ & 1,5 & 7,2 & 13,6 & 2,6 & 36,3 & 0,44 & $<0,0001$ \\
\hline & $\mathrm{dc}$ & 1,8 & 8,5 & 18,0 & 3,7 & 43,1 & 0,67 & $<0,0001$ \\
\hline & APHC & 2,5 & 67,0 & 255,0 & 53,6 & 80,0 & 0,65 & $<0,0001$ \\
\hline & $\mathrm{Fc}$ & 0,37 & 1,24 & 6,72 & 0,65 & 52,7 & 0,29 & 0,0014 \\
\hline
\end{tabular}

FLORESTA, Curitiba, PR, v. 48, n. 1, p. 123 - 132, jan. / mar.2108 


\begin{tabular}{ccccccccc}
\hline & $\mathrm{h} / \mathrm{d}$ & 0,27 & 0,55 & 1,42 & 0,22 & 41,0 & $-0,51$ & $<0,0001$ \\
\cline { 2 - 8 } & $\mathrm{Is}$ & 0,07 & 0,24 & 0,39 & 0,05 & 21,9 & $-0,08$ & 0,3643 \\
\cline { 2 - 8 } & $\mathrm{Ia}$ & 0,11 & 0,48 & 0,85 & 0,15 & 32,0 & 0,57 & $<0,0001$ \\
\cline { 2 - 8 } Índices de competição & IC1 & 0 & 0,67 & 4,41 & 0,90 & 133,6 & $-0,24$ & 0,0070 \\
\cline { 2 - 8 } & IC2 & 0 & 0,84 & 1,38 & 0,29 & 34,6 & 0,01 & 0,9175 \\
\cline { 2 - 8 } & IC3 & 0 & 0,82 & 4,05 & 0,85 & 102,8 & 0,32 & 0,0003 \\
\cline { 2 - 8 } & IC4 & 0 & 6,69 & 27,43 & 5,99 & 89,6 & $-0,54$ & $<0,0001$ \\
\cline { 2 - 8 } & IC5 & 0 & 1,10 & 5,49 & 1,15 & 104,0 & $-0,20$ & 0,0304 \\
\hline
\end{tabular}

Em que: Mín. = valor mínimo; Máx. = valor máximo; $\mathrm{S}=$ desvio-padrão; CV\% = coeficiente de variação; ${ }^{*}$ Coeficiente de correlação de Pearson ( $\rho)$ do IPAg $\left(\mathrm{cm}^{2}\right.$.ano $\left.{ }^{-1}\right)$ em função das variáveis dendrométricas, morfométricas e índices de competição. IPAd = incremento periódico anual em diâmetro, em cm; IPAg = incremento periódico anual em área transversal, $\mathrm{cm}^{2}$.ano ${ }^{-1} ; \mathrm{IPAg} \%=$ incremento periódico anual em área transversal percentual; $d=$ diâmetro à altura do peito, em $\mathrm{cm} ; d_{-5 t}=$ diâmetro no início do período, em $\mathrm{cm} ; d_{\mathrm{sc}}=$ diâmetro sem casca, em $\mathrm{cm} ; \mathrm{h}$ = altura total, em m; hic = altura de inserção de copa, em m; hc = altura comercial, em m; cc = comprimento de copa, em m; dc = diâmetro de copa, em m; APHC = área de projeção horizontal de copa, em m²; Fc = forma de copa; relação h/d; Is = índice de saliência; Ia = índice de abrangência; IC = índices de competição calculados: Basal Area Larger [IC1]; Glover e Holl I - [IC2]; Glover e Holl II - [IC3]; Lorimer [IC4]; Hegyi [IC5]; Rouvinen e Kuuluvainen [IC6], respectivamente.

As variáveis dendrométricas diâmetro (d) e suas variações, altura total (h) e altura de inserção de copa (hic) apresentaram correlações significativas com o IPAg (Tabela 2). Apesar de o diâmetro ter apresentado correlação positiva forte $(\rho=0,64 ; p<0,0001)$ com o IPAg, optou-se por utilizar o diâmetro no início do período analisado ( $\left.\mathrm{d}_{-5 \mathrm{t}}\right)(\rho=0,58 ; \mathrm{p}<0,0001)$, uma vez que, segundo Costa et al. (2016b), possibilita prognosticar o IPAg futuro em função de medidas realizadas no período atual.

A altura total $(\rho=0,50 ; p<0,0001)$ foi selecionada por ser uma variável de caracterização do sítio. Assim, pode ser dizer que o padrão de crescimento em altura das espécies ou de um povoamento é altamente dependente da combinação dos fatores genéticos e ambientais (sítio).

As variáveis morfométricas e os índices de competição, em sua maioria, apresentaram correlação significativa com o IPAg, exceto para o índice de saliência $(\rho=-0,08 ; p=0,3643)$ e para o índice de competição de Glover e Holl I (IC2) $(\rho=0,01 ; p=0,9175)$ (Tabela 2). Dentre os atributos de dimensão da copa, o diâmetro de copa (dc) foi a variável selecionada, pois apresentou a maior correlação de Pearson com a variável dependente em questão $(\rho=0,67 ; p<0,0001)$. Além do diâmetro da copa, a relação $\mathrm{h} / \mathrm{d}$ foi outra variável morfométrica selecionada, apesar de não ter apresentado maior correlação com o IPAg $(\rho=-0,51 ; p<0,0001)$.

Dentre os índices de competição analisados, o índice de Lorimer (IC4) apresentou a maior correlação ( $\rho$ $=-0,54 ; p<0,0001)$ com o IPAg. A forte correlação negativa observada para esse índice indica que o crescimento em área transversal de $O$. porosa decresce conforme aumentam as dimensões das suas árvores vizinhas e a competição sobre ela.

Além disso, diante das correlações positivas entre o incremento de O. porosa e suas variáveis dendrométricas e morfométricas selecionadas, verifica-se que, a medida em que a árvore cresce em diâmetro e altura e alonga seu diâmetro de copa, há um incremento também em área transversal e, consequentemente, em volume.

O modelo linear generalizado (MLG) para descrever o IPAg de O. porosa foi ajustado a partir das variáveis independentes selecionadas, sendo testandas as distribuiçõesNormal, Gamma e Gaussiana inversa e as funções de ligação de identidade $(\mu)$, logarítmica $(\ln \mu)$ e de potência $\left(\mu^{\lambda}\right)$. Por apresentar os menores valores de AIC e BIC, optou-se por utilizar o MLG com a distribuição Gamma e a função de ligação logarítmica (ln $\mu$ ) (Tabela 3). 
Tabela 3. Critérios de ajuste dos modelos lineares generalizados usados para descrever o incremento periódico anual em área transversal de $O$. porosa.

Table 3. Fit criteria of generalized linear models used to describe the annual periodic increment in basal area of $O$. porosa.

\begin{tabular}{|c|c|c|c|}
\hline MLG & F.L. & AIC & BIC \\
\hline \multirow[t]{3}{*}{ Normal } & $\mu$ & 995,8 & 1015,5 \\
\hline & $\ln (\mu)$ & 973,8 & 993,4 \\
\hline & $\mu^{\lambda}$ & 967,7 & 987,3 \\
\hline \multirow[t]{3}{*}{ Gamma } & $\mu$ & 925,6 & 945,2 \\
\hline & $\ln (\mu)$ & 924,1 & 943,7 \\
\hline & $\mu^{\lambda}$ & 930,7 & 950,3 \\
\hline \multirow[t]{2}{*}{ Gaussiana inversa* } & $\mu$ & 971,3 & 990,9 \\
\hline & $\ln (\mu)$ & 990,3 & 1010,0 \\
\hline
\end{tabular}

Em que: $\mathrm{MLG}=$ modelo linear generalizado; F.L. = função de ligação; $\mathrm{AIC}=$ Critério de Informação de Akaike; BIC = Critério de Informação de Bayesiano; $\mu=$ identidade; $\ln (\mu)=\operatorname{logarítmica} ; \mu^{\lambda}=$ potência com $\lambda=-1$. ${ }^{*} \mu^{\lambda}=$ não convergiu.

Os coeficientes de todas as variáveis selecionadas para compor o modelo foram significativos (Tabela 4). Assim, fica evidenciado que o crescimento da espécie é uma combinação dependente: da qualidade do sítio, expresso pela altura das árvores; da competição passada, expressa pelo diâmetro de copa; da estabilidade e vitalidade das árvores, expressa pela relação h/d; e da competição atual a qual a árvore está exposta, expressa pelo índice de Lorimer (IC4). Esse índice, independente da distância, demonstra relação biológica em explicar a influência da competição atual no crescimento de $O$. porosa, corroborando com sua utilização em florestas mistas e inequiâneas, devido à facilidade na determinação a campo e sua praticidade no uso.

Tabela 4. Coeficientes de regressão do modelo linear generalizado usados para descrever o incremento periódico anual em área transversal de $O$. porosa.

Table 4. Regression coefficients of generalized linear model used to describe the annual periodic increment in basal area of $O$. porosa.

\begin{tabular}{lllllll}
\hline Parâmetros & Coeficientes & Erro padrão & L.C. Wald 95\% & Wald $\chi^{2}$ & Pr $>\chi^{2}$ \\
\hline$\hat{\beta}_{0}$ (Intercepto) & 8,2736 & 1,3426 & 5,6422 & 10,9050 & 37,98 & $<0,0001$ \\
\hline$\hat{\beta}_{1}\left(\ln \left(\mathrm{d}_{-5 \mathrm{t}}\right)\right)$ & $-1,9857$ & 0,4186 & $-2,8062$ & $-1,1652$ & 22,50 & $<0,0001$ \\
\hline$\hat{\beta}_{2}(\mathrm{~h})$ & 0,1706 & 0,0327 & 0,1064 & 0,2347 & 27,13 & $<0,0001$ \\
\hline$\hat{\beta}_{3}(\mathrm{dc})$ & 0,0979 & 0,0278 & 0,0435 & 0,1523 & 12,42 & 0,0004 \\
\hline$\hat{\beta}_{4}(\mathrm{~h} / \mathrm{d})$ & $-3,4106$ & 0,7537 & $-4,8878$ & $-1,9334$ & 20,48 & $<0,0001$ \\
\hline$\hat{\beta}_{5}(\mathrm{IC} 4)$ & $-0,0561$ & 0,0129 & $-0,0814$ & $-0,0307$ & 18,79 & $<0,0001$ \\
\hline
\end{tabular}

Em que: $\hat{\beta}_{0} . \hat{\beta}_{5}=$ coeficientes de regressão estimados; L.C. $=$ limite de confiança; $\mathrm{d}_{-5 \mathrm{t}}=$ diâmetro no início do período analisado em cm; $\mathrm{h}=$ altura total em $\mathrm{m} ; \mathrm{dc}=$ diâmetro de copa em $\mathrm{m} ; \mathrm{h} / \mathrm{d}$ = razão entre a altura e o diâmetro; IC4 = índice de competição de Lorimer (IC4).

A análise gráfica (Figura 2a) mostra que os valores de incremento estimados pelo modelo selecionado são próximos aos valores observados, principalmente em árvores de menores diâmetros. A dispersão dos resíduos (Figura 1b) também comprova a eficiência do modelo em estimar o incremento periódico anual em área transversal. Nota-se que não há tendência dos resíduos ao longo da linha média, comprovando a eficiência do modelo para descrever o incremento periódico em área transversal. 

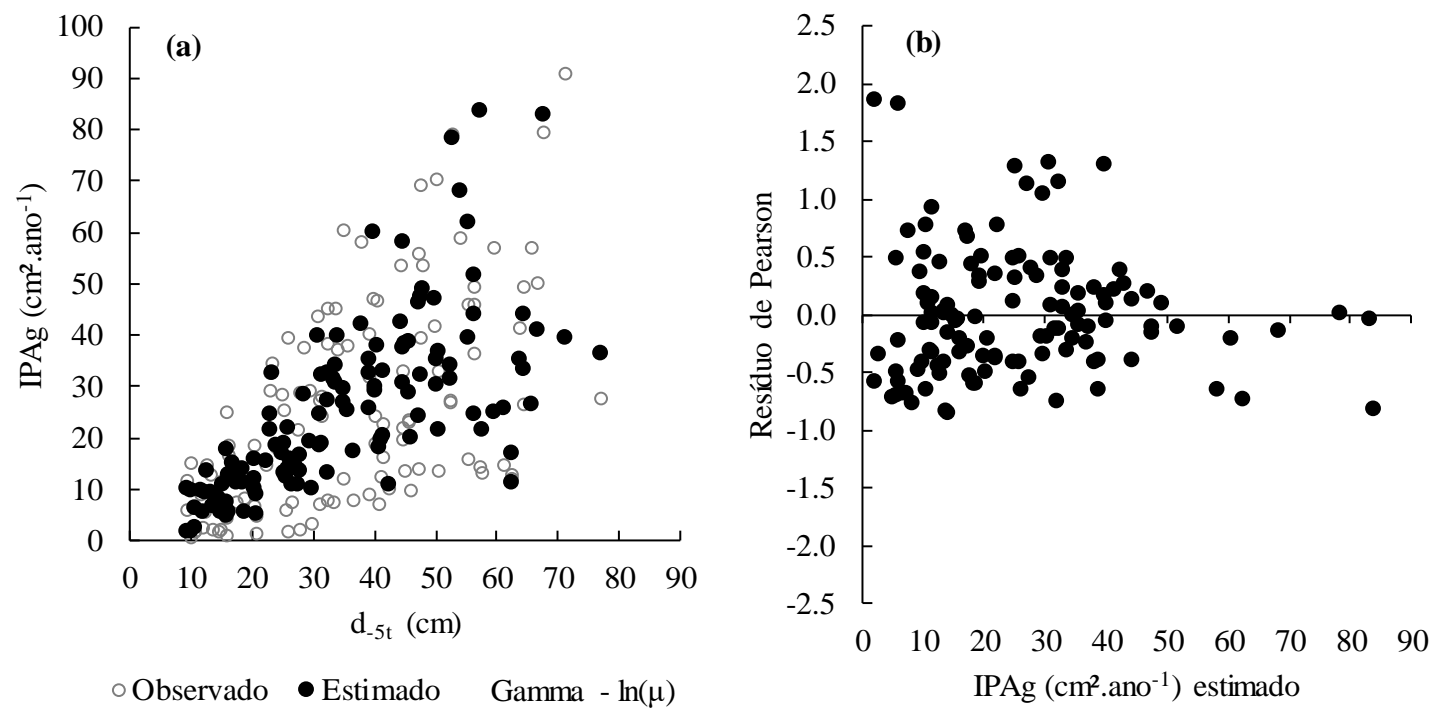

Figura 2. Dispersão dos valores observados e estimados (a); e resíduos de Pearson (b) do IPAg de O. porosa gerados pela MLG com a distribuição de Gamma e a função de ligação logarítmica

Figure 2. Dispersion of the observed and estimated values (a); and Pearson residuals (b) of $O$. porosa generated by MLG with Gamma distribution and link function logarithmic.

O modelo selecionado pela modelagem linear generalizada apresentou ajuste sem tendência na análise gráfica dos resíduos(Figura 2b). Embora o modelo possa ser utilizado para descrever o incremento de $O$. porosa em outros locais, é necessário sua validação. Além disso, o MLG selecionado possui vantagens em relação à modelagem linear pelo método dos mínimos quadrados ordinários quando não é observada estabilidade da variância ocasionada em dados não normais ou assimétricos, sendo necessária a transformação da variável resposta. Nesse caso, a MLG mostra-se mais flexível, dada a possibilidade de adequar os dados da variável resposta com o respectivo preditor linear por meio de uma função de ligação, proporcionando estimativas não enviesadas. Assim, nas estimativas obtidas do incremento e área transversal da $O$. porosa gerados pela técnica de MLG, foi possível a obtenção de coeficientes do modelo com realismo biológico, o que permite a interpretação adequada do modelo.

\section{DISCUSSÃO}

Os resultados de incremento encontrados neste trabalho corroboram com os de outros autores que estudaram taxas de crescimento para a espécie. Cubas et al. (2016) encontrou o mesmo valor de incremento $\left(0,43 \mathrm{~cm} \mathrm{ano}^{-1}\right)$ ao estudar o crescimento da espécie em uma Floresta Ombrófila Mista no município de Três Barras (SC). Os autores consideram esse crescimento expressivo quando comparado a demais espécies analisadas e relacionam esse valor com a capacidade ecológica da imbuia em se desenvolver em florestas com alta competição. Mattos et al. (2010) verificaram, por análise dendrocronológica, um incremento médio em diâmetro de $0,59 \mathrm{~cm} \cdot \mathrm{ano}^{-1} \mathrm{em}$ uma Floresta Ombrófila Mista em Caçador (SC), mesma região do presente estudo. Em plantios puros dessa espécie, onde se espera um maior crescimento quando comparado à floresta nativa, Santos et al. (2015) verificaram incremento de $0,61 \mathrm{~cm} \cdot$ ano $^{-1}$. Os autores comentam que diferenças no crescimento da espécie em condições naturais ou em povoamentos implantados podem ser decorrentes da variabilidade genética, das características ambientais do local ou mesmo das condições de competição presentes e passadas.

A seleção do diâmetro (d) no modelo gerado para descrever o incremento em área transversal de $O$. porosa é de suma importância, pois esta é uma variável que apresenta boa correlação com outras características da floresta, além de ser de fácil obtenção (ZHAO et al., 2014). Devido à sua influência no incremento, o dap foi selecionado para explicar o crescimento de espécies advindas de florestas naturais e plantadas, como nos trabalhos de Zimmermann et al. (2016) e Cunha et al. (2016).

Da mesma forma, a inclusão da variável altura total (h) no modelo de crescimento de imbuia é de suma importância, pois, de acordo com Campos e Leite (2013), a produtividade do sítio depende da qualidade ambiental da área, que se reflete diretamente no crescimento em altura das árvores dominantes. Apesar das dificuldades de determinação dessa variável em florestas naturais, a altura total tem sido selecionada para compor os modelos de

FLORESTA, Curitiba, PR, v. 48, n. 1, p. 123 - 132, jan. / marc. 2018.

Weber. V. P. et al.

ISSN eletrônico 1982-4688

DOI: $10.5380 /$ rf.v48 i1.54368 
incremento de espécies nativas por diversos autores, devido à sua alta correlação com o incremento em área transversal (CUNHA et al., 2016, COSTA et al., 2016b).

O diâmetro de copa é considerado uma variável importante na estimativa de outras variáveis da árvore e possui correlação positiva com o crescimento em diâmetro e, consequentemente, com o incremento em área transversal (ZIMMERMANN et al., 2016), além de ser uma variável capaz de prever o espaço vital futuro exigido para o desenvolvimento das árvores (CONDÉ et al., 2012). De acordo com Wink et al. (2012), as estimativas de biomassa da copa podem ser determinadas a partir das relações morfométricas. As dimensões da copa interferem na fisiologia da planta, podendo ser utilizadas como indicadores de vigor das árvores. Elas estão ligadas diretamente com a capacidade da árvore em competir por recursos (LEITE et al., 2012).

A relação h/d pode ser utilizada como indicativo do nível de competição e diretrizes de planos de desbaste. Ainda, segundo Durlo et al., (2004), o conhecimento das variáveis de copa e das relações morfométricas pode fornecer informações de espacialidade entre as árvores, ajudando a inferir sua estabilidade, produtividade e vitalidade.

De acordo com o gráfico de dispersão dos incrementos (Figura 2a), observa-se que árvores de maior dimensão incrementam mais em área transversal, pois esse incremento não depende somente da área incrementada, mas também do diâmetro inicial no período analisado. Assim, é esperado que árvores com maiores diâmetros apresentem maiores incrementos que árvores menores em um mesmo período.

\section{CONCLUSÕES}

- A modelagem linear generalizada com distribuição Gamma na variável dependente e função de ligação logarítmica $(\ln \mu)$ apresentou os melhores resultados quanto aos critérios de avaliação da performance preditiva do modelo. $\mathrm{O}$ uso dessa metodologia permite predições não enviesadas, com maior acuracidade $\mathrm{e}$ realismo biológico na interpretação dos coeficientes de regressão, quando comparados aos métodos convencionais com transformação na variável dependente.

- O modelo de incremento periódico anual em área transversal de árvores individuais de O. porosa foi descrito pelas variáveis de tamanho (diâmetro à altura do peito no início do período e relação h/d), dimensão de copa (diâmetro de copa), competição atual (índice de Lorimer) e sítio (altura).

- Esse modelo é uma importante ferramenta para a tomada de decisões no manejo racional dessa espécie. Podese simular cenários ótimos que maximizem a produção de árvores comerciais, além de beneficiar atividades ligadas ao planejamento, ordenamento e práticas silviculturais da espécie.

\section{AGRADECIMENTOS}

Os autores agradecem a M. Sc. Flávio Roberto Stefanello, M. Sc. Guilherme Boeira Ivanov e M. Sc. Cilmar Antônio Dalmaso por sua ajuda durante a medição dos dados. Além disso, agradecemos a Geraldo Busato, proprietário da empresa Laminados AB pelo apoio durante as medições. Agradecemos também a Dra. Bruna Denardin da Silveira e Dra. Maria Augusta Doetzer Rossot por permissão de coleta de dados complementares no Inventário Florestal Contínuo da Reserva Florestal EMBRAPA/ EPAGRI de Caçador (SC), e a Dra. Patrícia Póvoa de Mattos pela ajuda na identificação dos anéis de crescimento.

\section{REFERÊNCIAS}

ALVARES, C. A.; STAPE, J. L.; SENTELHAS, P. C.; GONÇALVES, J. L. M.; SPAROVEK, G. Köppen's climate classification map for Brazil. Meteorologische Zeitschrift, v. 22, n. 6, p. 711-728, 2013.

CAMPOS, J. C. C.; LEITE, H. G. Mensuração florestal: perguntas e respostas. 4.ed. Viçosa. MG: Universidade Federal de Viçosa, 2013. 605p.

CANETTI, A.; MATTOS, P. P.; BRAZ, E. M.; RICKENA, P.; PELLICO NETTO, S. Retrospective analysis of competition in a forest remnant: A case study of Podocarpus lambertii in the Araucaria Forest. Dendrochronologia, v. 40, p. 43-49, 2016.

CONDÉ, T. M.; LIMA, M. L. L.; TONINI, H.; LIMA NETO, E. M. Equações morfométricas das espécies andiroba (Carapa guianensis AUBL) e castanha-do-brasil (Bertholletia excelsa H. B. K.) em sistemas agroflorestais no município de Porto Velho. Rondônia. Revista Eletrônica Ambiente - Gestão e Desenvolvimento, v. 4, n. 1, p. $1-21,2012$. 
CORDEIRO, G. M.; DEMÉTRIO, C. G. B. Modelos lineares generalizados e extensões. Departamento de Estatística e Informática, UFRPE e ESALQ/USP, 2008. 392 p.

COSTA, E. A.; FINGER, C. A. G.; FLEIG, F. D. Influência da posição social nas relações morfométricas de Araucaria angustifolia. Revista Ciência Florestal, v. 26, p. 225-234, 2016a.

COSTA, E. A.; FINGER, C. A. G.; HESS, A. F. Modelagem do incremento em área transversal de árvores de crescimento livre de Araucaria angustifolia. Revista Brasileira de Biometria, v. 34, p. 522-532, $2016 \mathrm{~b}$.

COSTA, E. A.; FINGER, C. A. G.; HESS, A. F. Modelo de incremento em área basal para árvores de araucária de uma floresta inequiânea. Pesquisa Florestal Brasileira. v. 35, n. 83, p. 239-245, 2015.

CUBAS, R.; WATZLAWICK, L. F.; FIGUEIREDO FILHO, A. Incremento, ingresso, mortalidade em um remanescente de Floresta Ombrófila Mista em Três Barras, SC. Revista Ciência Florestal, v. 26, n. 3, p. 889-900, 2016

CUNHA, T. A.; FINGER, C. A. G.; HASENAUER, H. Tree basal area increment models for Cedrela. Amburana. Copaifera and Swietenia growing in the Amazon rain forests. Forest Ecology and Management, v. 365, p. 174$183,2016$.

DANIELS, R. F.; BURKHART, H. E.; CLASON, T. R. A comparison of competition measures for predicting growth of loblolly pine trees. Canadian Journal of Forest Research, v. 16, n. 6, p. 1230-1237, 1986.

DURLO, M. A.; SUTILI, F. J.; DENARDI, L. Modelagem da copa de Cedrela fissilis Vellozo. Revista Ciência Florestal, v. 14, p. 79-89, 2004.

FOX, J. Applied Regression Analysis and Generalized Linear Models, $3^{\circ}$ ed., Sage Publications Inc. 2015. $816 \mathrm{p}$.

INSTITUTO BRASILEIRO DE GEOGRAFIA E ESTATÍSTICA - IBGE. Manual Técnico da Vegetação Brasileira. 2a ed. Rio de Janeiro: IBGE. 2012. Disponível em: <http://www.ibge.gov.br/home/geociencias/recursosnaturais/vegetacao/manual_vegetacao.shtm>

KIERMAN, D. H.; BEVILACQUA, E.; NYLAND, R. D. Individual-tree diameter growth model for sugar maple trees in uneven-aged northern hardwood stands under selection system. Forest Ecology and Management, v. 256, p. $1579-1586,2008$.

LEITE, L. P.; ZUBIZARRETA-GERENDIAIN, A.; ROBINSON, A. Modeling mensurational relationships of plantation - growth loblolly pine (Pinus taeda L.) in Urugay. Forest Ecology and Management, v. 289, p. 455462, 2012.

LHOTKA, J. M. Examining growth relationships in Quercus stands: An application of individual-tree models developed from long-term thinning experiments. Forest Ecology and Management, v. 385, p. 65-77, 2017.

MATTOS, P. P. DE; OLIVEIRA, M. F.; AGUSTINI, A. F.; BRAZ, E. M.; RIVERA, H.; OLIVEIRA, Y. M. M. DE; ROSOT, M. A. D.; GARRASTAZU, M. C. Aceleração do crescimento em diâmetro de espécies da Floresta Ombrófila Mista nos últimos 90 anos. Pesquisa Florestal Brasileira, v. 30, n. 64, p. 319-326, 2010.

PAUlA, A. G. Modelos de regressão com apoio computacional. Instituto de Matemática e Estatística Universidade de São Paulo. 2013.

SANTOS, A. T. DOS; MATTOS, P. P. DE; BRAZ, E. M.; ROSOT, N. C. Determinação da época de desbaste pela análise dendrocronológica e morfométrica de Ocotea porosa (Nees \& Mart.) Barroso em povoamento não manejado. Revista Ciência Florestal, v. 25, n. 3, p. 699-709, 2015.

SAS INSTITUTE. The SAS system for Windows. Release 9.2. SAS Inst., Cary, NC. 2011.

WEISKITTEL, A. R.; HANN, D. W.; KERSHAW, J. A.; VANCLAY, J. K. Forest growth and yield modeling. Chichester: Wiley-Blackwell. 2011. 415 p.

WINK, C.; MONTEIRO, J. S.; REINERT, D. J.; LIBERALESSO, E. Parâmetros da copa e a sua relação com o diâmetro e altura das árvores de eucalipto em diferentes idades. Scientia Florestalis, v. 40, n.93, p. 57-67, 2012.

FLORESTA, Curitiba, PR, v. 48, n. 1, p. 123 - 132, jan. / marc. 2018

Weber. V. P. et al.

ISSN eletrônico $1982-4688$

DOI: $10.5380 /$ rf.v48 i1.54368 
ZHAO, J.; MAGUIRE, D. A.; MAINWARING, D.; WEHAGE, J.; KANASKIE, A. Thinning Mixed-Species Stands of Douglas-Fir and Western Hemlock in the Presence of Swiss Needle Cast: Guidelines Based on Relative Basal Area Growth of Individual Trees. Forest Science, v. 60, n.1, p. 191-199, 2014.

ZIMMERMANN, A. P. L.; COSTA, E. A.; SCHRODER, T.; FLEIG, F. D. Modelagem do incremento diamétrico de Pinus taeda em função de variáveis da copa e índices de competição. Floresta (UFPR. Impresso),v.46,p.115$122,2016$. 